\title{
An unusual presentation of female urethral leiomyoma
}

\section{Neville D Perera ${ }^{1}$, Lakshman Senanayake ${ }^{4}$, V Hasanthi Vithana ${ }^{2}$ and Ruchira Fernando ${ }^{3}$}

(Index words: Diagnostic approach, histology, urethral and bladder reconstruction)

\section{Abstract}

Leiomyoma of the urethra is a rare clinical entity. We report an unusual presentation of this tumour, which led to a diagnostic and surgical dilemma. The patient was a 16year old female who presented with a labial mass which was palpable abdominally. Imaging methods and laparoscopy demonstrated a well defined soft tissue mass arising from the pelvis, without any obvious involvement of the urinary tract. The tumour $(8 \times 10 \mathrm{~cm})$ was completely excised with reconstruction of the bladder and urethra. Histology confirmed a cellular leiomyoma.

\section{Introduction}

Urethral leiomyoma is rare and only about 50 cases have been reported in literature. This benign smooth muscle tumour often presents as a midline extramucosal vaginal mass, which is often mistaken for a urethral diverticulum, fibroid or prolapse. We report an unusual presentation of a large lateral tumour, which led to a dilemma in diagnosis and surgical approach.

${ }^{1}$ Urological Surgeon, ${ }^{2}$ Research Assistant, Department of Urology, and ${ }^{3}$ Pathologist, Department of Pathology, National Hospital of Sri Lanka; ${ }^{4}$ Obstetrician and Gynaecologist, Castle Street Hospital (Teaching) for Women, Colombo. Correspondence: NDP, e-mail: <nevi603@sltnet.lk> (Competing interests: none declared). Received 15 June 2004 and accepted 20 August 2004. 


\section{Case report}

A 16-year old unmarried girl presented with a history of progressively enlarging painless lump at the vulva for 6 months. Other than the discomfort it caused during walking, there were no symptoms. Examination of the external genitalia revealed a visible and palpable lump under the right labium majus, which was firm, spherical and nontender. The urethral meatus and vaginal opening were distorted and deviated to the opposite side. Bimanual palpation per rectum revealed the tumour to be extending as a mobile pelvic mass.

Abdominal ultrasound and CT scan demonstrated a well defined pelvic soft tissue mass lying between the bladder and the rectum, displacing the uterus and vagina towards the left side. It extended superiorly up to the lumbosacral articulation and inferiorly to the right labial fold. There was no sign of infiltration into the surrounding structures (Figure 1).

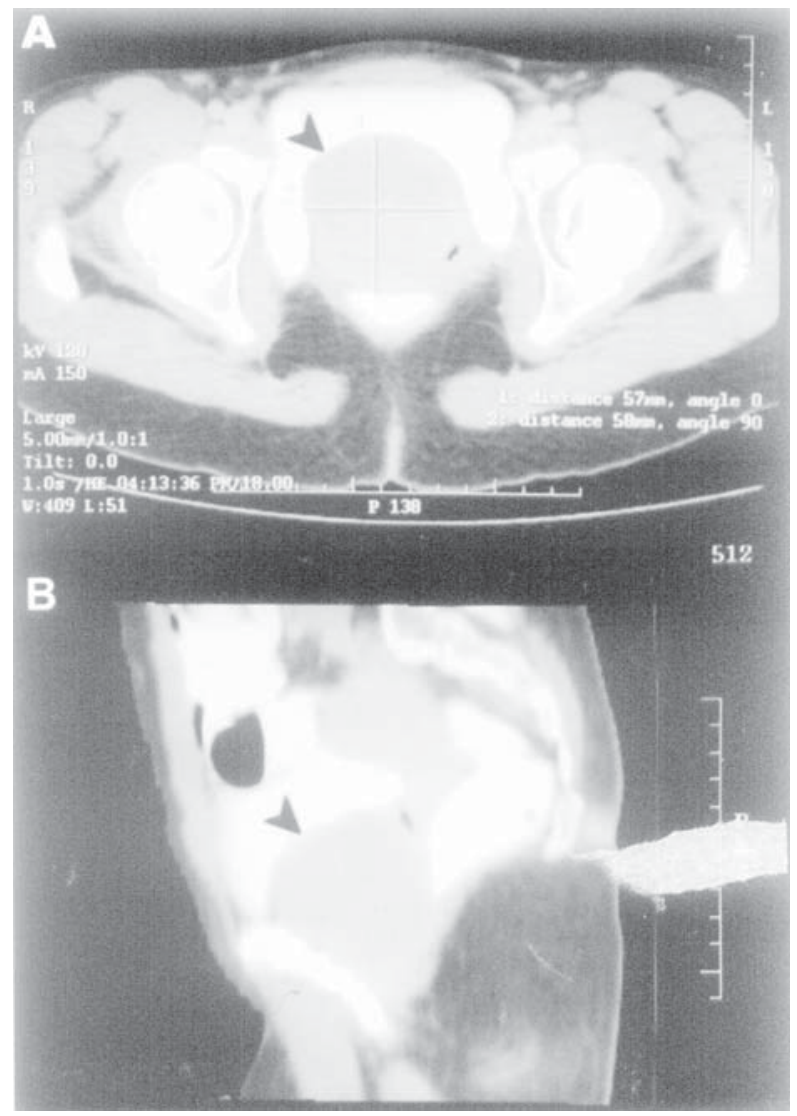

Figure 1. CT scan shows the leiomyoma (arrow), A lying between the bladder and rectum; $B$ shows the extent of tumour.

A differential diagnosis of an ovarian neoplasm or retroperitoneal teratoma was contemplated. Tumour markers were negative. Radiologically there was no involvement of the urinary tract. Pre-operative ureteric stenting was attempted to prevent operative trauma, and was successful only on the right side. Urethral mucosa was intact and appeared normal during cystourethroscopy.
Diagnostic laparoscopy showed a solid mass protruding postero-laterally between the bladder and the uterus.

Trans-abdominal exposure showed a large $(8 \times 10 \mathrm{~cm})$ retroperitoneal tumour attached to the posterior urethral wall. Complete excision of the mass was done, which resulted in a partial urethrectomy. The bladder and the urethra were reconstructed with tabularised anterior bladder wall. Presence of the J stent was a useful guide to ureteric orifices. The recovery was uneventful with good urinary continence.

Macroscopically the tumour tissue was soft, tan, with a uniform cut surface. Microscopy revealed a cellular tumour with interlacing bundles of spindle cells with abundant eosinophilic cytoplasm and elongated blunt-ended nuclei with minimal cellular atypia. This appearance was consistent with the cellular urethral leiomyoma (Figure 2).

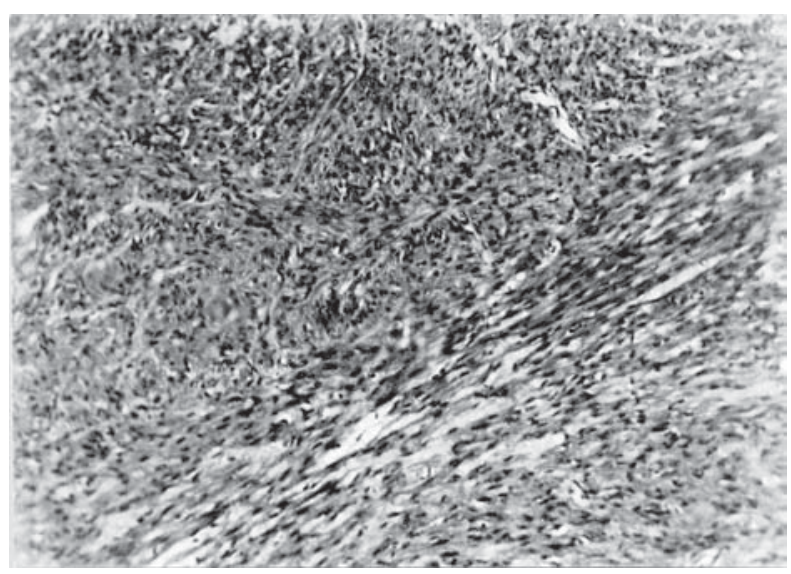

Figure 2. Typical histological features of leiomyoma of the urethra with interlacing bundles of spindle cells with minimal cellular atypia. A section stained with $H$ \& $E(x 40)$.

\section{Discussion}

Leiomyoma of urethra is rare, with a strong female preponderance. The exact aetiology and pathogenesis remain unclear, though an endocrine dependency is suspected as several cases of urethral leiomyoma were reported to enlarge during pregnancy and regress after delivery [1-3].

The morphological features of urethral leiomyoma are similar to its counterparts elsewhere. They tend to grow as isolated, encapsulated, oval or spherical masses of varying diameter, ranging from few millimeters to several centimetres, with the largest reported up to now measuring $8 \mathrm{~cm}$ [3]. The most common site is the posterior wall of the urethra. The common clinical presentations include periurethral or vaginal mass, dysuria, dyspareunia, haematuria, and rarely obstructive urinary symptoms $[1,2,4-6]$. In the presence of a urethral mass it may be confused with urethral diverticulum, caruncle, papilloma and carcinoma.

The recommended treatment is complete local excision through the vagina. Large tumours as in the present case, need a trans-abdominal approach with urethral and 


\section{Case reports}

bladder neck reconstruction. Prognosis is excellent as recurrence is very rare and malignant transformation is not known to occur [7].

A labial mass with intra-abdominal extension, as in the present case, is an extremely rare presentation which can lead to a diagnostic dilemma. This case illustrates the importance of surgical expertise for urethral reconstruction.

\section{References}

1. Fry M, Wheeler JS Jr, Mata JA, Culkin DJ, St Martin E, et al. Leiomyoma of the female urethra. Journal of Urology 1988; 140: 613-4.

2. Alvarado-Cabrero I, Candanedo-Gonzalez F, SosaRomero A. Leiomyoma of the urethra in a Mexican woman: a rare neoplasm associated with the expression of estrogen receptors by immunohistochemistry. Archives of Medical Research 2001; 32: 88-90.

3. Peterson RO. Urologic Pathology. 2nd ed. Philadelphia: JB Lippincott, 1992: 419.

4. Deka PM, Rajeev TP. Leiomyoma of the female urethra: A case report. Urology International 2003; 71: 224-5.

5. Cornella JL, Larson TR, Lee RA, Magrina JF, KammererDoak D. Leiomyoma of the female urethra and bladder: Report of twenty three patients and review of the literature. American Journal of Obstetrics and Gynecology 1997; 176: $1278-85$.

6. Dasan JCR, Rao K, Nalini V. Leiomyoma of the female urethra - a clinical curiosity. International Journal of Gynecology and Obstetrics 1989; 28: 381-3.

7. Lake MH, Kossow AS, Bokinsky G. Leiomyoma of the bladder and urethra. Journal of Urology 1981; 125: 742-3. 